\title{
Caracterización de los casos de abuso sexual valorados en los servicios de urgencias y consulta externa de una institución hospitalaria de primer nivel en el Departamento del Cauca, 2007 - 2015
}

Characterization of sexual abuse cases valued in the emergency services and outpatient consultation of a firstlevel hospital institution in the department of Cauca, 2007 - 2015

Gloria Cerón-Hernández' orcid.org/ 0000-0003-1551-7988

Sandra Roa-Torres ${ }^{2}$ orcid.org/0000-0002-2271-6101

Mercedes Salcedo-Cifuentes ${ }^{3 *}$ orcid.org/ 0000-0002-9681-6893

1 Organización Internacional de las Migraciones OIM - Instituto Colombiano de Bienestar Familiar ICBF- Unidades Móviles. Pasto, Colombia

2 Litigante independiente. Villa Rica - Cauca, Colombia

3 Facultad de Salud, Universidad del Valle. Cali, Colombia

Fecha de recepción: Agosto 17 - 2016 Fecha de revisión: Febrero 21 - $2016 \quad$ Fecha de aceptación: Agosto 11 - 2017

Cerón-Hernández G, Roa-Torres S, Salcedo-Cifuentes M. Caracterización de los casos de abuso sexual valorados en los servicios de urgencias y consulta externa de una institución hospitalaria de primer nivel en el Departamento del Cauca, 2007 - 2015. Univ. Salud. 2017;19(2):226-236. DOI: http://dx.doi.org/10.22267/rus.171902.85

\section{Resumen}

Introducción: El abuso sexual afecta a millones de niños, niñas y adolescentes e impacta en su salud física y mental. Objetivo: Caracterizar los casos de abuso sexual valorados en los servicios de urgencias y consulta externa de una institución hospitalaria de primer nivel del Departamento del Cauca entre 2007 al 2015. Materiales y métodos: Estudio descriptivo, retrospectivo de víctimas de abuso sexual atendidas en consulta externa/urgencias. Se seleccionaron variables temporales, sociodemográficas de la víctima/agresor y del contexto donde ocurrió el hecho. El análisis se realizó en Epi Info. Se calcularon medidas de tendencia central, dispersión, proporciones y razones. Con el test de Fischer se evaluó la relación entre variables. Resultados. El 77\% de las víctimas fueron mujeres, 23\% hombres, en edades entre los 2-16 años, el 100\% eran estudiantes. El acto fue cometido por un solo agresor; en el 93,0\% fue perpetrado por conocidos, de los cuales $42,9 \%$ eran familiares. Conclusiones. A pesar de las medidas de control y regulación, los resultados sugieren que el abuso sexual tiene lugar a edades muy tempranas, el agresor es casi siempre un conocido frecuentemente con vínculo consanguíneo, lo cual le facilita la perpetuación del hecho hacia el interior del núcleo familiar a través del engaño, el chantaje o la amenaza.

Palabras clave: Niño; abuso sexual; vulnerabilidad; víctima. (Fuente: DeCS, Bireme).

\begin{abstract}
Introduction: Sexual abuse affects millions of children and adolescents and it impacts on their physical and mental health. Objective: To characterize the cases of sexual abuse valued in the emergency services and external consultation of a hospital institution of first level in the department of Cauca between 2007 and 2015. Materials and methods: A descriptive, retrospective study of victims of sexual abuse attended in external consultation/emergencies was made. Temporal and sociodemographic variables of the victim/aggressor and the context where the event occurred were selected. The analysis was done in Epi Info. Measures of central tendency, dispersion, proportions and reasons were calculated. The relationship between variables was assessed by using the Fischer test. Results: $77 \%$ of the victims were women, $23 \%$ men, at ages between 2 to 16 years and $100 \%$ were students. The act was committed by a single aggressor. Besides, in $93.0 \%$ of the cases, the act was perpetrated by
\end{abstract}


acquaintances, of whom $42.9 \%$ were family members. Conclusions: Despite the control and regulation measures, the results suggest that sexual abuse takes place at very early ages. The aggressor is almost always a commonly known person with consanguineous bond, which facilitates the perpetuation of the act towards the family nucleus through deceit, blackmail or threat.

Keywords: Children; sexual abuse; vulnerability; victims. (Source: DeCS, Bireme).

\section{Introducción}

El abuso sexual infantil es un problema universal con efectos graves para toda la vida, es una forma de violencia a la infancia(1). La Organización Mundial de la Salud (OMS) define el abuso sexual infantil como la situación en donde: "se involucrar al niño en actividades sexuales que no llega a comprender totalmente, a las cuales no está en condición de dar consentimiento informado, o para las cuales está evolutivamente inmaduro y tampoco puede dar consentimiento, o en actividades sexuales que trasgreden las leyes $o$ las restricciones sociales"(2), el término incluye además una serie de actividades como: "la relación sexual, intento de coito, contacto oral-genital, caricias de los genitales directamente o través de la ropa, el exhibicionismo o la exposición de los niños a la actividad sexual o la pornografía de adultos, y el uso de los niños para la prostitución o la pornografía"(3).

El tema es complejo y difícil de estudiar, las estimaciones varían ampliamente dependiendo del país, las definiciones utilizadas, el tipo de abuso, la extensión de la cobertura y la calidad de los datos, entre otros(1-5), sin embargo, la violencia sexual está presente en todo el ciclo vital humano, en todas las clases socioeconómicas, y casi en todos los países con diferencias en la magnitud(4).

Sumner et al.(6), indicaron que la exposición a la violencia durante la infancia puede aumentar la vulnerabilidad a una amplia gama de problemas de salud mental y física, que van desde la depresión y el embarazo no deseado, a las enfermedades cardiovasculares, diabetes y enfermedades de transmisión sexual, incluida la infección por el Virus de Inmunodeficiencia Humana (VIH).
Los estudios han encontrado que los actos más violentos cometidos contra los niños, niñas y adolescentes son perpetrados por personas que forman parte del entorno inmediato de la víctima ${ }^{7}$. Así un informe de Snyder en el $2000^{8}$ con datos del departamento de justicia de Estados Unidos mostró que la mayoría de las agresiones físicas reportados por la policía contra los niños menores de 6 fueron cometidos por alguien conocido de la víctima (81\%). En este mismo sentido los reportes de estadísticas canadienses ${ }^{(9)}$, indicaron que las agresiones a los menores de 18 años por lo general no implican el uso de un arma.

Aguilar y Salcedo(10), reportaron que estrategias como el chantaje o el engaño, eran usadas por el agresor como una forma fácil para acercarse al menor y lograr la inclusión del componente sexual en la relación con este, enmascarando el abuso. En estas formas de chantaje tuvieron mediación el dinero o algún bien material prometido a la víctima. Resultados semejantes fueron presentados por Spraggon en el 2000(11).

Este tipo de hechos violentos llegan con mayor frecuencia a servicios de salud, donde los menores son traslados por los padres o parientes. Sin embargo, muy pocos se oficializan ante el sistema penal, por lo cual, las estadísticas que develen la verdadera magnitud del problema tienen altos subregistros. En este sentido, este estudio pretendió caracterizar los casos de abuso sexual valorados en los servicios de urgencias y consulta externa de una institución hospitalaria de primer nivel del Departamento del Cauca, en el periodo comprendido del 2007 al 2015, con el fin de mejorar el conocimiento que se tiene con relación a los casos de abuso sexual atendidos en los servicios de salud. 


\section{Materiales y métodos}

Se realizó un estudio descriptivo de tipo retrospectivo del 2007 al 2015 en el que se incluyeron todos los casos de abuso sexual infantil, atendidos en los servicios de urgencias y consulta externa de una institución hospitalaria de primer nivel perteneciente a la red de salud pública del Departamento del Cauca. El contexto donde se llevó a cabo el estudio, fue un municipio con predominio de población afrodescendiente, predomina la etnia negra, los núcleos familiares se caracterizan por un ausentismo de los padres quienes laboran en los cañaduzales y los menores quedan al cuidado de amigos u otros familiares cercanos ${ }^{(12)}$.

Las fuentes de información fueron: la base de datos de radicación de casos atendidos en los servicios de urgencias, consulta externa y las historias clínicas. El estudio recopiló datos temporales y variables consideradas en los tres primeros niveles del Modelo Teórico Ecológico.

En el nivel individual se describieron las variables sociodemográficas de la víctima y el estado de alicoramiento del supuesto agresor. En el nivel relacional, el vínculo con el supuesto agresor, la forma como este sometió a la víctima al momento del hecho y cómo la indujo a que no lo denunciara. En el nivel comunitario, se consideró el contexto en el cual se llevó a cabo el $\operatorname{abuso}^{(4)}$.

Para el procesamiento de la información se diseñó una hoja electrónica en la cual se realizó la captura de los datos y después se transfirió al paquete estadístico Epi Info para su análisis. A las variables cuantitativas se les calculó medidas de tendencia central (media, moda y mediana) así como de dispersión (desviación estándar) y a las cualitativas las proporciones y razones. A través del Test de Fischer, se exploró la relación entre las variables considerando un valor de $\mathrm{p} \leq 0,05$ como estadísticamente significativo.

\section{Consideraciones éticas}

El proyecto fue catalogado sin riesgo ya que se recurrió a recolectar información a partir de fuentes secundarias. Fue aprobado por el Comité de Ética de la Universidad Libre Seccional Cali, siguiendo las normas de la Resolución No. 008430 de 1993 del Ministerio de Salud de Colombia y contó con la aprobación de las directivas del hospital fuente de información.

\section{Resultados}

Un total de 30 casos fueron incluidos en el estudio, 21 atendidos en el servicio de urgencias y 7 en consulta externa. Su distribución por año se observa en la Figura 1.

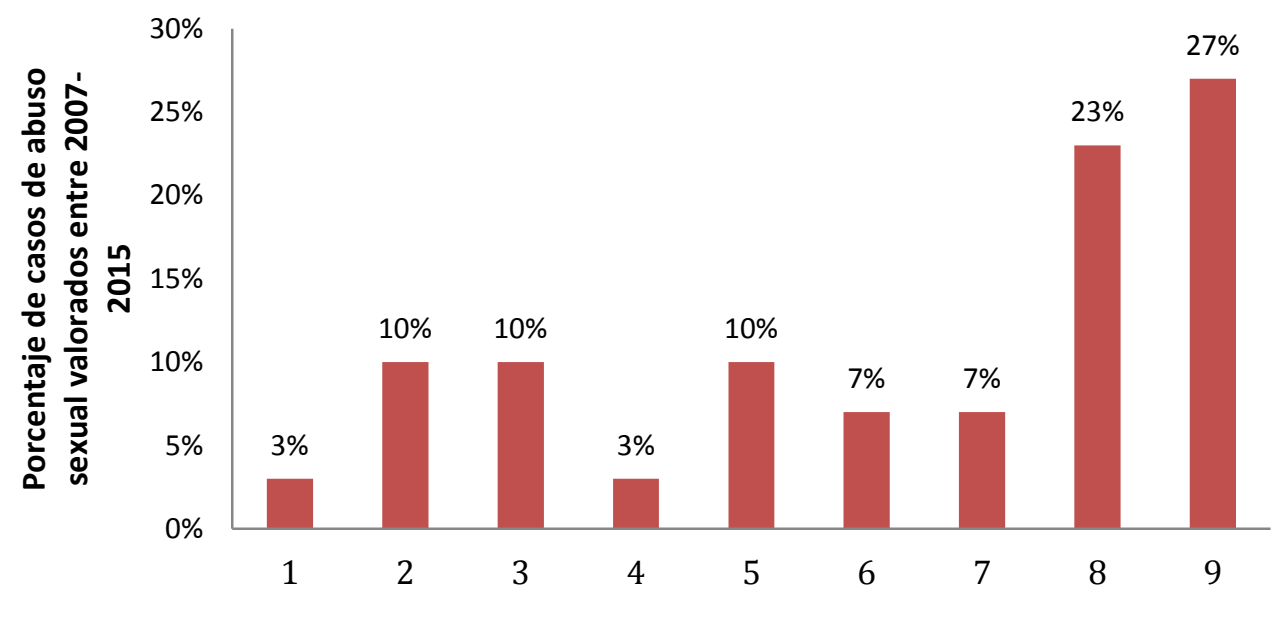

Figura 1. Distribución por año de los casos de delito sexual incluidos en el estudio 
Según día de la semana, la mayoría de los abusos ocurrieron entre los días miércoles y viernes (21 casos) y 8 lunes, martes, sábado o domingo. Con relación a la hora del hecho, se observó la ocurrencia de 3 casos en el horario vespertino por cada caso en el horario nocturno, 17 de las historias clínicas revisadas no contaban con este dato.

En el nivel individual, 23 casos fueron mujeres y 7 hombres. Con relación a los casos valorados en el servicio de urgencias, la edad mínima de la víctima fue de 2 años y la edad máxima de 16, con una razón de 3 casos en mayores de 10 años por cada menor de diez años. Los siete casos valorados en el servicio de consulta externa correspondieron a menores entre 11 y 13 años. No se encontraron diferencias estadísticamente significativas entre los menores y mayores de 10 años según servicio de atención (Test de Fischer, $\mathrm{p}=0,2474$ ) (Figura 2).

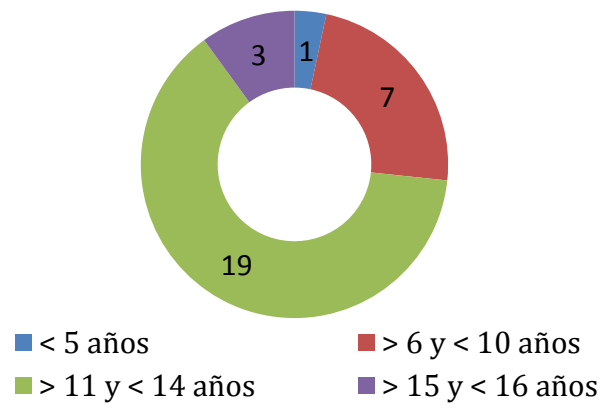

Figura 2. Distribución de la variable edad en los casos incluidos en el estudio

En concordancia con el rango de edad prevalente, el $97 \%$ de los menores se encontraban escolarizados, 9 en primaria y 20 en secundaria. El 90\% de los usuarios llegaron a ser atendidos a través de su EPS del régimen subsidiado, mientras que en el $10 \%$ de los casos no se registró su vinculación al sistema de seguridad social.

Con respecto al nivel relacional, en el 7,0\% $(2 / 30)$ de los casos el hecho fue cometido por un desconocido y en el 93,0\% (28/30) fue un conocido de la víctima, dentro de estos el 42,9\% $(12 / 28)$ un familiar. No se presentaron casos con agresores múltiples y 100\% fueron perpetrados por hombres. En dos de los 30 casos, según su relato, se presentó recurrencia del hecho por el mismo agresor, uno fue un amigo de la familia y en el otro el tío.

Según Código Penal Colombiano todos los casos fueron catalogados como de abuso sexual, es decir relaciones sexuales de adultos, tocamientos y caricias. Seis casos fueron accesos carnales en menores de 14 años (tres cometidos por amigos y tres por el tío), 2 accesos carnales en mayores de 14 años fueron consumados por desconocidos y dos actos sexuales, uno cometido por el padre del menor y otro por el tío. No se observaron diferencias estadísticamente significativas entre el tipo penal y el vínculo con el agresor (Test de Fischer, $\mathrm{p}=0$,1957) (Tabla 1).

Tabla 1. Tipo relación de la víctima con el agresor y tipo de abuso

\begin{tabular}{lcccc}
\hline Vínculo & $\mathbf{N}^{\circ}$ Casos & $\mathbf{\%}$ & $\begin{array}{c}\mathbf{N}^{\circ} \text { Abusos } \\
\text { sexuales* }\end{array}$ & Otros $^{\mathbf{E}}$ \\
\hline Amigo & 16 & 53 & 13 & 3 \\
Tío & 9 & 30 & 5 & 4 \\
Padre & 3 & 10 & 2 & 1 \\
Desconocido & 2 & 7 & & 2 \\
Total & $\mathbf{3 0}$ & $\mathbf{1 0 0}$ & $\mathbf{2 0}$ & $\mathbf{1 0}$ \\
\hline $\begin{array}{l}\text { Según Código Penal: }{ }^{*} \mathrm{y}^{£} \text { incluyó accesos carnales abusivos, accesos carnales } \\
\text { y actos sexuales }\end{array}$ &
\end{tabular}

Según vínculo familiar con el agresor, el hecho se cometió en el entorno intrafamiliar en 12 de los 30 casos ( 2 de las víctimas fueron hombres y 10 mujeres) y 18 en el extrafamiliar ( 6 hombres y 12 mujeres). No se observaron diferencias estadísticamente significativas entre ellos (Test de Fischer, $p=0,4192$ ).

De los 30 casos estudiados, dos reflejaron en el resumen de los hechos que el agresor se encontraba con algún nivel de estado de alicoramiento:

"adolescente de 12 años de edad que ingresa en compañía de su madre por el servicio de urgencias para ser atenido por presunto abuso sexual del menor por parte de su padre borracho, relaciona maniobras sexuales tales como besos y caricias, a madre es quien refiere haber sorprendido a su pareja abusando de su hija."

Relato de la víctima atendida en 2014. 
"Menor que ingresó por servicio de urgencias en compañía de su tía, refiere ser abusado sexualmente. Usuaria consulta 10 días después de la ocurrencia del hecho. Agresor el padre quien se encontraba bajo efectos del alcohol."

Relato de víctima atendida en 2015.

Los relatos del hecho mostraron que en 14 de los 30 casos, el agresor sometió a la víctima a través del engaño, la amenaza o con el uso de la fuerza.
En dos se usó tanto la fuerza como el engaño y en uno la amenaza y el engaño. Por otra parte, en seis de los casos la denuncia estuvo dentro de las primeras 72 horas y tan solo en dos casos esta fue hecha mucho más allá de las 72 horas. En estos no fue posible analizar el porqué del silencio post-asalto dado que la descripción del hecho no dio elementos para ello. Un resumen de estos hallazgos se presenta en la Tabla 2.

Tabla 2. Hallazgos en los resúmenes que sugieren sometimiento e inducción al silencio a través del uso de la fuerza, amenaza, chantaje o engaño

\begin{tabular}{ll}
\hline $\begin{array}{c}\text { No relato-año de } \\
\text { reporte }\end{array}$ & \multicolumn{1}{c}{ Resumen del hecho } \\
\hline $\mathbf{1 - 2 0 0 8}$ & "escolar que ingresa en compañía de su madre, refiere la \\
& madre que su hija fue abusada por un amigo de la familia \\
& hace aproximadamente tres horas, la menor dice que el \\
& agresor le dio dinero a cambio de un beso, pero luego empezó \\
a manosearle el cuerpo, por lo cual empezó a gritar pidiendo & \\
& auxilio" \\
& "paciente que ingresa al servicio de urgencias caminando por \\
$\mathbf{2 - 2 0 0 9}$ & sus propios medios en compañía de la madre la cual \\
& manifiesta que hubo abuso sexual por parte de un tío quien \\
& frecuenta la casa de la familia muy de seguido,... "ese señor \\
& quiso tener relaciones por la fuerza conmigo, que si no me \\
& dejaba le iba hacer daño a mi mamán"
\end{tabular}

3-2010

4-2011

5-2012

6-2013

7-2013

8-2014 "paciente que ingresa por área de urgencias, en compañía de la madre la cual informa que su hijo requiere atención medica por que hace algunos días, aproximadamente 15 o 18 días, fue víctima de abuso sexual por parte del tío, no había dicho nada por miedo"

"niña que ingresa por servicio de urgencias en compañía de un familiar,... "un amigo intento abusar de mí, empezó a tocarme y a besarme me amenazó varias veces con lastimarme si no me dejaba,..."

"me encontraba jugando futbol y cuando se terminó el partido nos quedamos hablando con un amigo, luego el amenazó con golpearme y empezó a darme besos y agarrarme la cola, yo lo empuje y salí corriendo..."

"mi tío llego a la casa y subió a mi cuarto se quedó acompañándome a ver televisión después empezó a jugar conmigo me empezó a darme besos y a tocarme los genitales entonces yo lo empecé a quitar de mí y me amenazó con hacerme castigar de mi mamá porque le estaba desobedeciendo, así había pasado varias veces, pensé que mi mami me castigaría por desobedecerle a mi tío, no sabía que eso era malo"

"niño que ingresa en compañía de un familiar, víctima de abuso sexual hace aproximadamente media hora, situación que se presenta bajo amenaza y engaño"

"adolescente de 12 años de edad que ingresa caminando por sus propios medios acompañado de un familiar (abuela), adecuado vestir, colabora con entrevistador, en estado consciente sin signos de ingesta de alcohol o sustancias alucinógenas, refiere haber sido abusado por un amigo bajo engaños y por la fuerza"

\begin{tabular}{cll} 
Sometimiento & $\begin{array}{c}\text { Inducción al silencio con relación a } \\
\text { la hora de denuncia }\end{array}$ \\
\hline Chantaje & $\begin{array}{l}\text { Ninguno, denuncia dentro de las } \\
\text { primeras } 72 \text { horas }\end{array}$ & \\
\hline
\end{tabular}

Fuerza Chantaje

Sin dato Chantaje, denuncia dos semanas después del hecho

$\begin{array}{ll}\text { Amenaza } & \begin{array}{l}\text { Ninguno, denuncia dentro de las } \\ \text { primeras } 72 \text { horas }\end{array} \\ \text { Amenaza } & \begin{array}{l}\text { Ninguno, denuncia dentro de las } \\ \text { primeras } 72 \text { horas }\end{array}\end{array}$

Amenaza Miedo, no se especifica hace cuánto es víctima, pero la situación es recurrente

Amenaza y engaño

Engaño y fuerza
Ninguno, denuncia dentro de las primeras 72 horas

Ninguno, denuncia dentro de las primeras 72 horas 
9-2014

10-2014

11-2015

12-2015

13-2015

14-2015 "escolar femenina que ingresa para atención por medicina general, vestida en adecuadas condiciones caminando por sus propios medios y orientada en espacio y tiempo, refiere haber sido víctima de abuso sexual, por individuo conocido cercano de la familia, la situación se presentó en la vivienda de la adolescente mediante engaños el día 5 de septiembre..."

"menor de edad que ingresa por servicios de urgencia en compañía de un primo, el familiar informa que la niña fue abusada por el tío con el uso de la fuerza, manifiesta no haber asistido a valoración médica una vez sucedido el hecho por temor..."

"niña que ingresa por urgencias y refiere...mi tío me llevó a su casa a pasear, cuando llegamos no había nadie en la casa y comenzó a acariciarme y besarme por la fuerza, entonces grite y pude salir. La madre refiere que la situación se había presentado hace como dos semanas aproximadamente no se había consultado antes por que no tenía conocimiento de lo ocurrido, cuando empezó a ver cambios de actitud de la niña se preocupó, entonces le pregunto a la niña que le estaba pasando y la niña le refirió que su tío le hizo caricias sexuales y que si la niña avisaba le haría daño".

"la paciente ingresa por servicio de urgencias, con adecuado vestir, caminando por sus propios medios en estado de conciencia, manifiesta que su tío le hizo caricias por todo el cuerpo aludiendo haberle dado dinero y que por ello se tenía que dejar, eso ocurrió el día de ayer".

"menor...manifiesta ser abusada por un familiar, hechos ocurridos aproximadamente hace 3 horas, la menor le da a conocer a la madre lo sucedido, ..."mi tío me tomó por la fuerza y comienza a acariciarme los genitales y empieza a ejercer maltrato físico para que me calle..."

"menor...refiere haber sido abusada sexualmente, sin mencionar o identificar al agresor, los hechos se llevaron a cabo en zona poblada de árboles por donde transitaba la adolescente donde fue tomada por la fuerza hace aproximadamente un mes...".
Engaño Sin dato, la víctima es atendida más allá de 72 horas, aproximadamente 11 días después

Fuerza Temor

Engaño y fuerza Amenaza

Chantaje Ninguno, denuncia dentro de las primeras 72 horas

Fuerza

Fuerza

Fuerza

Sin dato, la víctima es atendida más allá de 72 horas, aproximadamente 30 días después.
En el nivel comunitario se identificó el contexto en el cual ocurrieron los casos. Se observó una razón de 3 abusos sexuales cometidos en la vivienda de la víctima o el agresor en relación con otro escenario (Figura 3).

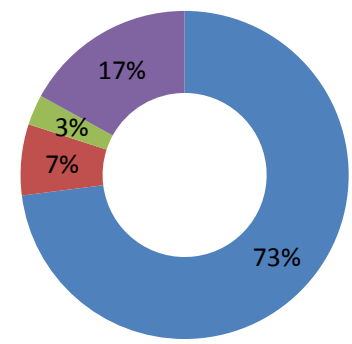

- Vivienda víctima
Espacio diversión

- Vivienda agresor

- Campo abierto

Figura 3. Lugar donde ocurrieron los hechos

Al cruzar el lugar donde ocurrió el abuso sexual y el sexo de la víctima se observó que 22 de los casos se perpetuaron en la residencia de habitación del menor, 16 fueron contra niñas. De igual forma, aquellos que ocurrieron en la residencia del agresor, el menor ofendido fue una niña (2/2), en esta relación no se observaron diferencias estadísticamente significativas (Test de Fischer, $p=0$,0568). Por último, con relación a las variables del nivel social, ninguno de los casos fue puesto en conocimiento del Instituto Colombiano de Bienestar Familiar o ante la Fiscalía.

\section{Discusión}

El abuso sexual en menores de edad es un problema social cada vez más reconocido que impacta negativamente en la salud y el bienestar de ellos, de su familiar y de toda la comunidad ${ }^{13}$. Estudios de corte histórico, sociológico y epidemiológico han documentado casos 
ocurridos en distintas regiones de Europa y América, develando las situaciones sociales de abuso sexual y las modalidades del agresor para encubrir el hecho y mantener la cronicidad del mismo, así como antecedentes de agresión sexual en los victimarios ${ }^{(14)}$. Aunque se sabe que hay un subregistro muy alto(15), se reconoce por las estadísticas oficiales que el mayor número de víctimas son del género femenino. Sin embargo, muchos casos cometidos contra la libertad e integridad sexual masculina son encubiertos por la sociedad o no son denunciados por las víctimas para evitar que el hecho lo relacionen con un posible comportamiento homosexual(16). Según resultados de varias investigaciones, denunciar un abuso sexual es denunciar una experiencia homosexual forzada(17).

Si bien no existe límite de edad para ser víctima de este tipo de hechos violentos, de acuerdo a lo expuesto en el Modelo Teórico Ecológico, la edad temprana en las mujeres puede constituirse en un factor de riesgo. Según una revisión de varias investigaciones que consideraron este factor, la prevalencia mundial de victimización sexual en la niñez estuvo alrededor del $27 \%$ en niñas y aproximadamente del $14 \%$ en niños(18). De igual forma los datos de los sistemas judiciales y de los centros de atención de crisis por violación en Chile, Estados Unidos, Malasia, México, Nueva Guinea y Perú, muestran que aproximadamente entre un tercio a dos de todos los casos de las víctimas de agresión sexual y tráfico de mujeres con fines de explotación sexual se presentan en menores de 15 años(19). Para muchos expertos, esa esfera mundial de violencia sexual contra niñas y adolescentes está muy ligada a las pautas de comportamiento y modelos machistas, de patriarcados arraigados tradicionalmente, que tienden a creer que tienen el derecho de controlar el cuerpo y la sexualidad de las mujeres(20).

En Colombia, según informe del observatorio del delito de la Policía, durante el 2013 cerca del $35 \%$ de los niños, niñas y adolescentes abusados sexualmente se encontraban entre los 12 y 14 años. Los hechos fueron cometidos principalmente contra el género femenino con una tasa participativa del $85,5 \%(21)$. Estos resultados fueron similares a los de GonzálezForteza et al.(22), en México, quienes encontraron como población más vulnerable a las mujeres.

Por otra parte, Ramos-Lira et al.(23), concluyeron que el rango de edad más prevalente estaba entre los 9 y los 13 años, resultados consistentes con los de este estudio. En general, los estudios coinciden que la población más vulnerable son los menores de edad y del género femenino.

Por otra parte, la relación entre el grado de instrucción de la presunta víctima y la ocurrencia del delito sexual es compleja. Un bajo nivel educativo es, sin embargo, el factor que se asocia con mayor constancia con la perpetración y el padecimiento de hechos denunciados(24). Por el contrario, un nivel más alto de educación pareciera actuar como un factor protector, ya que las personas con un mayor nivel educativo, reportan en menor proporción hechos de violencia sexual(25). Este es un hallazgo que no se puedo confirmar en este estudio, dado que el $100 \%$ de las víctimas incluidas en este estudio fueron menores de edad y el nivel de educación fue una variable ausente en las historias clínicas.

Con relación al no registro de datos sociodemográficos en las historias clínicas, es un hallazgos consistente en otros estudios como el del Curia et al.(26), quienes reportaron su ausencia en una gran proporción, especialmente en lo que respecta a información sobre educación, ocupación, estado civil y edad.

Es importante tener en cuenta las condiciones de vida de las víctimas y victimarios, por lo general los casos de violencia sexual se presentan en familias que viven en condiciones de pobreza, este es un factor de riesgo muy frecuente en la literatura sobre violencia intrafamiliar y dentro de esta, la violencia sexual, pero aclaran los expertos que no es un fenómeno que deba verse exclusivamente asociado con los hogares pobres, que por el contrario está presente en todos los estratos socioeconómicos y si bien hay resultados de investigaciones que demuestran una fuerte asociación, este puede tener un sesgo de visibilización entre clases sociales ${ }^{20}$. Además, en Colombia, hay poca evidencia que demuestre 
cómo este tipo de violencia afecta de manera diferenciada a los distintos grupos socioeconómicos, y la forma como la pobreza incide en esta ${ }^{27}$. Sin embargo, para los expertos aún no hay resultados definitivos ni concluyentes. Es así cuando el escenario de violencia sexual, en el cual existe un proveedor único o principal de los ingresos económicos del núcleo familiar y éste ha sido el agresor sexual, la decisión de denunciar el hecho y romper la relación durante el proceso judicial es menguada, tanto por el riesgo inmediato de la caída drástica de medios de subsistencia para la familia, como por la utilización de esta herramienta por parte del agresor para chantajear y terminar manipulando en muchas ocasiones la conducción y decisión durante las audiencias y la intervención de la comisaría(27). En este estudio en particular los datos de vinculación al sistema de seguridad social de las víctimas, dieron evidencia que este fue un delito que se cometió en hogares que pertenecían a estratos socioeconómicos 1 y 2, es decir personas con un ingreso económico $\leq$ a un S.M.L.M.V.

Un análisis y discusión del grupo de variables individuales, plantea que el consumo de alcohol y sustancias psicoactivas, son factores que se presentan como una constante en los casos de violencia sexual y se citan con frecuencia como factores de riesgo asociados(18). Por otra parte, un meta-análisis que reunió los resultados de 11 estudios encontró que el consumo de alcohol se asociaba con un incremento de 4,6 veces el riesgo de exposición a hechos de violencia sexual. En estudios transversales provenientes de diferentes países de ingresos bajos y medianos han mostrado que los hombres que abusan del consumo de alcohol tienen un riesgo 1,6 a 4,8 veces mayor de ejercer violencia hacia el interior del núcleo familiar, en especial violencia sexual(18). Este es un dato que rara vez recolectan los profesionales asistenciales y que se evidenció en dos de los casos incluidos en el estudio a través de los relatos de las víctimas. Luego vale la pena resaltar la importancia de documentar muy bien la narración que realice la víctima de cómo ocurrieron los hechos.
Se destaca que las víctimas fueron niños, niñas y adolescentes, en donde el agresor resultó tener un vínculo cercano, un familiar o amigo, y es quizá una de las principales razones por la cual este tipo de delito no es informado a las autoridades competentes. En este grupo etario, la gran mayoría de los casos se mantiene mediante una coerción del abusador más o menos explícita y de represión emocional que puede incluir además elementos de amenaza física, acrecentando los temores a las consecuencias negativas y conllevando al silencio del menor(14).

Cuando el agresor es un familiar o un amigo del núcleo familiar, por lo general suelen tener mayor y mejor acceso al menor. Por tanto, existen mayores oportunidades de iniciar y continuar cualquier tipo de abuso. Vale la pena resaltar, que con frecuencia la dinámica del abuso inicia mediante estrategias de seducción, sobornos o amenazas e intimidación por parte del adulto conocido/familiar, sin necesariamente usar la fuerza física; de esta forma el abusador establece un vínculo con el niño o la niña creando un secreto entre los dos que lo protege de la denuncia(28). Estos resultados estuvieron presentes en los casos analizados en el presente estudio, en donde la inducción al silencio estuvo presente con el uso de la amenaza e intimidación. En menores de edad, el abuso sexual es intrafamiliar con frecuencias que oscilan entre el $65 \%$ y $85 \%$; en este ambiente de riesgo, la mayor proporción de agresores corresponde a un conocido familiar y dentro de estos al padre biológico con una prevalencia del 25\%(29). De aquí que el contexto de ocurrencia del evento en la mayoría de los casos (97\%) se trata de un lugar habitualmente conocido y frecuentado por el pequeño como es el sitio de residencia de la víctima (entre $28 \%$ y $43 \%$ de los casos) o del agresor (entre $21 \%$ y $34 \%$ de los casos).

La literatura indica que el abuso sexual intrafamiliar en menores de edad es considerablemente diferente de otras formas de violencia sexual, que las niñas son más propensas que los varones a ser víctimas; tiende a ocurrir con mayor frecuencia y durante un período más largo que el abuso sexual 
extrafamiliar; los actos causan mayor daño emocional y físico a la víctima; comienza a una edad más temprana; pueden ocurrir con otras formas de maltrato (por ejemplo, abuso físico) y a menudo los niños abusados y sus familias son desconocidos para las autoridades de protección infantil(20). Los resultados de este estudio son muy semejantes a lo expuesto y no difieren de los reportes en Colombia, en donde según la Policía Nacional, el lugar de ocurrencia de los hechos de violencia sexual en menores de edad ocurre en el $39,42 \%$ de los casos en la vivienda de las víctimas, el $28 \%$ en vías públicas y en el $15 \%$ dentro de la vivienda del agresor(21).

Tanto en las agresiones sexuales donde se involucra un agresor bajo los efectos del alcohol como en las que no, se comparten muchas características, pero al mismo tiempo existen algunas diferencias marcadas de acuerdo a la edad de la víctima. Por ejemplo, las agresiones sexuales que implican el consumo de alcohol son más probables entre hombres y mujeres que no se conocen bien y suelen ocurrir en las fiestas o en los bares. Este consumo puede perjudicar la capacidad de tomar decisiones, regular los afectos y controlar la impulsividad(31). El consumo general de alcohol podría estar relacionado con el asalto sexual de muchas maneras. Sin embargo, en este estudio el número de casos en los que se logró identificar agresores bajo efectos del alcohol fueron muy pocos. Valdría la pena explorar esta variable de manera más sistemática para evaluar si en el contexto local el abuso sexual si tiene como factor de riesgo el abuso del consumo de alcohol o si por el contrario el delito se está cometiendo con plena consciencia. Esto se refuerza con los resultados de Baltieri y Guerra(32), quienes indicaron que el consumo de alcohol y los problemas asociados con su uso no fueron diferentes entre los delincuentes sexuales seriales y los no seriales.

\section{Conclusiones}

Llama la atención que a pesar de todas las medidas de control y regulación contra este tipo de delito establecidas tanto a nivel nacional como internacional, las características de su ocurrencia (tiempo, lugar y persona) son constantes y comparables con estudios históricos; tienen patrones comportamentales muy parecidos, por no decir constantes. El grupo de agresores más representativo es un conocido amigo de la familia o un familiar, por lo cual, los estudios no deben limitarse solo a explorar las causas precipitantes o predisponentes, o la recurrencia del evento, entre otras características, sino también identificar la dinámica y organización familiar que posibilitan el escenario propicio para el desarrollo de este tipo de hechos violentos. Estos son espacios considerados "espacios privados familiares" que definitivamente están favoreciendo el encubrimiento y la impunidad y los médicos asistenciales junto con el equipo de trabajo interdisciplinario que deben atender a las víctimas, según marco legal en Colombia, Resolución 0459 del 2012(33), están en la obligación de recolectar suficientes datos que permitan hacer un análisis integral con el fin de definir la mejor estrategia de protección para los menores abusados.

\section{Agradecimientos}

Al Alcalde y Personero del Municipio donde se llevó a cabo la investigación, así como a las directivas y funcionarios del hospital fuente de información, por su colaboración y apoyo.

\section{Conflicto de intereses}

Los autores confirman que no tienen ningún conflicto de interés.

\section{Referencias}

1. Heisler K. Department Of Health \& Human Services. In Child maltreatment 2014. U.S. Department of Health \& Human Services. Washington: 2001. p. 17-50.

2. World Health organization. Guidelines for medicolegal care for victims of sexual violence. Child Sexual abuse. Geneva: WHO; 2003. Disponible en: http://apps.who.int/iris/bitstream/10665/42788/1/9 24154628X.pdf

3. Putnam FW. Ten year research update review: Child sexual abuse.J Am Acad Child Adolesc Psychiatry. 2003;42:269-78.

4. Krug EG, Mercy JA, Dahlberg LL, Zwi AB. The world report on violence and health. Lancet. 2002;360(9339):1083-8.

5. Bassani DG, Palazzo LS, Beria JU, Gigante LP, Figueiredo $\mathrm{AC}$, Aerts DR, et al. Child sexual abuse in southern 
Brazil and associated factors: A population based study. BMC Public Health. 2009;9:133-44.

6. Sumner SA, Mercy AA, Saul J, et al. Prevalence of sexual violence against children and use of social services seven countries, 2007-2013. MMWR Morb Mortal Wkly Rep. 2015;64(21):565-9.

7. Pinheiro PS. Worl Report on Violence Against Children. 2006. (Citado en julio 30 del 2016). Disponible en https://www.unicef.org/lac/full_tex(3).pdf

8. Howard N. Snyder. Bureau of Justice Statistics. Sexual Assault of Young Children as Reported to Law Enforcement: Victim, Incident, and Offender Characteristics. 2000. (Citado 30 de jul. 2016). Disponible

http://www.bjs.gov/content/pub/pdf/saycrle.pdf

9. Statistics Canada. Child and Youth Victims of Policereported Violent Crime, 2008. 85F0033M, no. 23. (Citado en julio 30 del 2016). Disponible en http://www.statcan.gc.ca/pub/85f0033m/2010023/p art-partie1-eng.htm.

10. Aguilar A, Salcedo-Cifuentes M. Caracterización de la ocurrencia de la violencia sexual en jóvenes entre 10 a 19 años. Cali. 2001-2003. Colombia Médica; 39(4):35663.

11. Spraggon C. Violencia sexual en Bogotá. Centro de Investigacones Sociojurídicas (CIJUS), Facultad de Derecho, Universidad de los Andes, Bogotá; 2000.

12. República de Colombia. Municipio de Villa Rica. Informe de gestión. Vigencia Fiscal 2009. Disponible en http://www.villarica-cauca.gov.co/apc-aafiles/66353037636261386533303637323963/INFOR ME_GENERAL.pdf

13. Abeid M, Muganyizi P, Massawe S, Mpembeni R, Darj E, Axemo P. (2015). Knowledge and attitude towards rape and child sexual abuse - a community-based cross-sectional study in Rural Tanzania. BMC Public Health. (Citado 12 de abril 2017). Disponible en https://www.ncbi.nlm.nih.gov/pmc/articles/PMC4419 $483 /$.

14. Vigarello G. Historia de la violación, siglos XVI-XX, Ediciones Universitat de Valencia, Madrid; 1998. pp2324.

15. León G. Del encubrimiento a la impunidad. Diagnóstico sobre violencia de género, Ecuador, 1989- 1992. Quito: CEIME Ediciones; 1995. pp 45-47.

16. Peluffo SM. Servicios de Salud para Víctimas/Sobrevivientes de Violencia Sexual: Buenas Prácticas. MÉXICO. Informe 2010. Disponible en www.ipas.org/ /media/Files/Ipas\%20Publications/Ip asCAMexico2010.ashx

17. Chavez R, Rivera-Rivera L, Angeles-Llerenas A, DíazCerón E, Allen-Leigh B, Lazcano E. Factores del abuso sexual en la niñez y la adolescencia en estudiantes de Morelos, México. Rev. Saúde Pública. 2009;43(3):506514.

18. OMS y OPS: Comprender y abordar la violencia contra las mujeres. Violencia sexual. (Citado 18 mar. 2016). Disponible

en http://apps.who.int/iris/bitstream/10665/98821/1/ WHO_RHR_12.37_spa.pdf?ua=1
19. Organización Mundial de la Salud. Informe mundial sobre la violencia: Resumen. Washington: OMS; 2002. (Citado en abril 18 del 2016). Disponible en http://www.who.int/violence_injury_prevention/viole nce/world_report/es/summary_es.pdf.

20. Contreras JM, Bott S, Guedes A, Dartnall E. Violencia sexual en Latinoamérica y el Caribe: análisis de datos secundarios. Iniciativa de Investigación sobre la Violencia Sexual. 2010. (Citado 20 de may. 2016). Disponible en: http://www.oas.org/dsp/documentos/Observatorio/v iolencia_sexual_la_y_caribe_2.pdf.

21. Colombia, Consejo Superior de la Judicatura. Observatorio del delito. Observatorio penal de delitos sexuales contra niños niñas y adolescentes en Colombia. Informe Ejecutivo . Junio 2015. [Citado junio 20 de 2016] Disponible en: https://www.google.com.co/url?sa=t\&rct=j\&q=\&esrc= s\&source=web\&cd=7\&cad=rja\&uact $=8 \& v e d=0$ ahUKEw iJmaK2ycPVAhUK6SYKHbAmBjwQFghQMAY\&url=http s\%3A\%2F\%2Fwww.ramajudicial.gov.co\%2Fdocument s\%2F1545778\%2F7429328\%2F1.\%2BINFORME\%2B EJECUTIVO\%2B\%2BOBSERVATORIO\%2BDE\%2BDELI TOS\%2BSEXUALES\%2BNNA.docx\%2F5897f048-04d44921-9a4d-

374e3959b18b\&usg=AFQjCNHtaqMkf0X2Mh8rOhfaAS AveGuemg

22. González-Forteza C, Ramos-Lira LE, Vignau-Brambila LE, Ramírez-Villarreal C. El abuso sexual y el intento suicida asociados con el malestar depresivo y la ideación suicida de los adolescentes. Salud Mental. 2001;24(6):16-25.

23. Ramos-Lira L, Saldívar-Hernández G, Medina-Mora ME, Rojas-Guiot E, Villatoro-Velázquez J. Prevalencia de abuso sexual en estudiantes y su relación con el consumo de drogas. Salud Pública México. 1998;40(3):221-33.

24. OMS y OPS: Prevención de la violencia sexual y violencia infligida por la pareja contra las mujeres Qué hacer y cómo obtener evidencias. 2010. (Citado 20 de junio. 2016). Disponible en http://apps.who.int/iris/bitstream/10665/44810/1/9 789275316351_spa.pdf.

25. Olivares E, Inchaustegui T. Modelo Ecológico para una vida libre de violencia de género. Comisión Nacional para Prevenir y Erradicar la violencia Contra las Mujeres. 2011. (Citado 24 de marzo. 2016). Disponible en:

https://www.gob.mx/cms/uploads/attachment/file/7 9360/MoDecoFinalPDF.pdf

26. Kuria M, Omondi l, Olando Y, Makenyengo M, Bukusi D. Is Sexual Abuse a Part of War? A 4-Year Retrospective Study on Cases of Sexual Abuse at the Kenyatta National Hospital, Kenya. J Salud Pública África . 2013;4(1):e5.

27. Pineda J, Otero L. Género, violencia intrafamiliar e intervención pública en Colombia. Revista de Estudios Sociales. 2004;17:19-31.

28. Almonte Carlos, Insunza Carla, Ruiz Cecilia. Abuso sexual en niños y adolescentes de ambos sexos. Rev. chil. neuro-psiquiatr. 2002;40(1):22-30. 
29. Pinheiro PS. Informe Mundial sobre la violencia contra los niños y niñas. Ginebra: Naciones Unidas; 2006.

30. Miller L. Sexual offences against children: Patterns and motives. Aggression and Violent Behavior. 2013; 18: 506-519.

31. Abbey A, Clinton-Sherrod AM, McAuslan P, Zawacki T, Buck PO. The relationship between the quantity of alcohol consumed and the severity of sexual assaults committed by college men.J Interpers Violence. 2003;18(7):813-33.

32. Baltieri D, Andrade A. Comparing serial and nonserial sexual offenders: alcohol and street drug consumption, impulsiveness and history of sexual abuse. Rev. Bras. Psiquiatr. 2008; 30( 1 ): 25-31

33. República de Colombia. Ministerio de Salud y la Protección Social. Resolución 0459 de 2012. Protocolo y modelo de atención integral en salud para víctimas de violencia sexual. (Citado en enero 30 de 2016). Disponible en https://www.minsalud.gov.co/sites/rid/Lists/Bibliote caDigital/RIDE/DE/DIJ/Resolucion-0459-de-2012.PDF 